\title{
Clinical usefulness of T1-weighted MR cholangiography with Gd-EOB-DTPA for the evaluation of biliary complication after liver transplantation
}

\author{
Chang Jin Lim*, Kwangpyo Hong*, Jeong-Moo Lee, Eui Soo Han, Suk Kyun Hong, \\ YoungRok Choi, Nam-Joon Yi, Kwang-Woong Lee, and Kyung-Suk Suh
}

\author{
Department of Surgery, Seoul National University College of Medicine, Seoul, Korea
}

\begin{abstract}
Backgrounds/Aims: Biliary complications continue to be the major morbidity and mortality causes following living donor liver transplantation (LT). Endoscopic retrograde cholangiopancreatography (ERCP) has been performed to identify the biliary leakage source. However, this can lead to retrograde cholangitis and pancreatitis, and is not sufficient to diagnose bile leakage from cuts' surface. This study aimed to describe the use of T1-Weighted Magnetic Resonance (MR) Cholangiography with Gd-EOB-DTPA (Primovist) examination for evaluating the bile duct complication following LT. Methods: From March 2012 to December 2018, 869 adult LT were performed at the Seoul National University Hospital. Forty-three recipients had undergone MR Cholangiography with Gd-EOB-DTPA. We reviewed these cases with their clinical outcomes and described the utility of the MR cholangiography with Gd-EOB-DTPA. Results: In radiologic examinations performed in the patients suspected of bile duct complication, $95 \%$ had bile leakage and stricture. Cut surface leakage was diagnosed in two cases, and biliary leakage from the anastomosis site was diagnosed in the others. Most patients with leakage had undergone percutaneous drainage and ERCP, which was performed to evaluate the bile secretion function of the hepatocytes. There was no contrast-enhanced bile duct image in one case with severe rejection, and it might have been related to hepatocyte secretary dysfunction. Conclusions: T1-Weighted MR Cholangiography with Gd-EOB-DTPA 40-minute delay examination is a feasible and safe non-invasive procedure for identifying biliary leakage sites. (Ann Hepatobiliary Pancreat Surg 2021;25:39-45)
\end{abstract}

Key Words: Gd-EOB DTPA MRI; Liver transplantation; Biliary complication

\section{INTRODUCTION}

Bile duct complications are still a pitfall and have a significant morbidity and mortality risk. Following liver transplantation (LT), leakages, obstructions, strictures, and stones have been observed in $9-30 \%$ of large clinical series of LT recipients. $^{1-4}$

Even with biliary complications, long-term survival can be expected when treated with appropriate intervention. The rapid diagnosis and appropriate intervention of the bile duct complication resolve biliary complication and enable long-term survival of the patients and grafts. The existing diagnostic method is to perform T-tube cholangiography in case of a history of T-tube, or examine the structure or leakage through endoscopic retrograde cholangiopancreatography (ERCP). With the recent development of surgical techniques, most liver transplant centers in Korea do not usually insert T-tube into the biliary tract during operation. ${ }^{5-7}$

However, these methods may increase the pressure in the bile duct due to the invasive procedure immediately after transplantation, and in the case of complications such as a minor leakage, can promote iatrogenic complications. Moreover, pancreatitis or cholangitis can occur because of the procedure.

Non-invasive diagnostic tools have been developed for biliary complications following a hepatobiliary surgery. Hepatobiliary scan (diisopropyl iminodiacetic acid [DISIDA])

Received: July 16, 2020; Revised: August 10, 2020; Accepted: August 12, 2020

Corresponding author: Jeong-Moo Lee

Department of Surgery, Seoul National University College of Medicine, 101 Daehak-ro, Jongno-gu, Seoul 03080, Korea

Tel: +82-2-2072-2817, Fax: +82-2-766-3975, E-mail: lulu5050@naver.com

*Chang Jin Lim and Kwangpyo Hong contributed equally to this work as co-first authors.

Copyright (C) 2021 by The Korean Association of Hepato-Biliary-Pancreatic Surgery

This is an Open Access article distributed under the terms of the Creative Commons Attribution Non-Commercial License (http://creativecommons.org/ censes/by-nc/4.0) which permits unrestricted non-commercial use, distribution, and reproduction in any medium, provided the original work is properly cited. Annals of Hepato-Biliary-Pancreatic Surgery • pISSN: 2508-5778 - elSSN: 2508-5859 
was performed to obtain a functional image of bile excretion. ${ }^{89}$ Alternatively, studies related to the diagnosis of biliary complications using magnetic resonance cholangiopancreatography (MRCP) have been reported. The MRCP revealed stricture and leakage following transplantation and the biliary leakage site could be identified in case of general resection. ${ }^{10-14}$

However, as the conventional magnetic resonance image (MRI) is severely blunted and moved, the image quality is too poor, it is often impossible to accurately identify the bile duct structure.

Recently, liver MRI using hepatobiliary contrast media such as gadoxetic acid (Gd-EOB-DTPA, Primovist, BayerSheringPharma, Berlin, Germany) have been used for the evaluation of various lesions and liver function. However, as the peak hepatic enhancement with gadoxetic acid is usually reached at 20-40 minutes following the injection, there may be a time gap between the dynamic phase imaging and the hepatobiliary phase imaging (HBPI), more clear and accurate bile duct images can be obtained when half of Gd-EOB DTPA is secreted through the kidney and the other half via the bile duct. Therefore, many centers usually perform MR Cholangiography with Gd-EOB-DTPA for donor biliary evaluations as well as to diagnose hepatocellular carcinoma.

The 20-min delayed image of Gd-EOB DTPA has an advantage of better visualization of the bile duct than with conventional MRCP. Recently, we included a protocol called "MR (magnetic resonance) cholangiography with Gd-EOBDTPA 40 min delay (MR pancreaticobiliary fistula protocol)" to evaluate leakage or stricture of the bile duct. The drainage pattern of the bile can be visualized more clearly; additionally, the synthetic function of the liver can be evaluated indirectly. This study examined the usefulness of MRCP with Gd-EOB-DTPA 40 min delay imaging protocol in LT.

\section{MATERIALS AND METHODS}

\section{Study design}

From March 2012 to December 2018, 869 adult living donor liver transplantations (LDLTs) were performed at the Seoul National University Hospital (SNUH). Among those, 43 recipients had undergone MRI pancreaticobiliary protocol. We reviewed the 43 cases with their clinical out- comes. This study was approved by the Institutional Review Board (IRB) of the SNUH (number "2007-028-1139").

Following an LT, MR Cholangiography with Gd-EOBDTPA was performed in cases suspected of biliary complication, such as when biloma was observed during fol-

Table 1. Demographics of the recipient who underwent Gd-EOB DTPB MRI after LT

\begin{tabular}{|c|c|}
\hline & $\mathrm{n}=43$ \\
\hline Age (years) & $51 \pm 12.9$ \\
\hline Gender (male-female) & $34: 9$ \\
\hline Bodyweight (kg) & $65.2 \pm 10.6$ \\
\hline Height $(\mathrm{m})$ & $166.5 \pm 6.64$ \\
\hline BMI $\left(\mathrm{kg} / \mathrm{m}^{2}\right)$ & $65.2 \pm 10.6$ \\
\hline \multicolumn{2}{|l|}{ Diagnosis } \\
\hline HBV LC & $19(44.2 \%)$ \\
\hline $\mathrm{HCV} \mathrm{LC}$ & $4(9.3 \%)$ \\
\hline Alcoholic LC & $14(35.6 \%)$ \\
\hline Autoimmune LC & $1(2.3 \%)$ \\
\hline Chronic rejection (retransplantatin) & $2(4.7 \%)$ \\
\hline Etc & $3(6.9 \%)$ \\
\hline \multicolumn{2}{|l|}{ Graft type } \\
\hline Right liver (living) & $41(95.3 \%)$ \\
\hline Left liver (living) & $1(2.3 \%)$ \\
\hline Whole liver (desceased) & $1(2.3 \%)$ \\
\hline \multicolumn{2}{|l|}{ Type of bile duct anastomosis } \\
\hline Duct-to duct & $35(81.4 \%)$ \\
\hline Hepatico-jejunostomy & $8(18.6 \%)$ \\
\hline $\begin{array}{l}\text { Date on Gd-EOB DTPA MRI } \\
{[\text { Postoperative day, median (range)] }}\end{array}$ & $36(9-2662)$ \\
\hline \multicolumn{2}{|l|}{ Causes of Gd-EOB DTPA MRI exam } \\
\hline Hyperbilirubinemia & $8(18.6 \%)$ \\
\hline Drain color change & $11(25.6 \%)$ \\
\hline Incidental Biloma on $\mathrm{CT}^{*}$ & $10(23.3 \%)$ \\
\hline ER visit abdominal pain & $14(32.5 \%)$ \\
\hline \multicolumn{2}{|l|}{ Findings of MRI leakage protocol } \\
\hline No leakage & $3(6.9 \%)$ \\
\hline Sitricture & $4(9.3 \%)$ \\
\hline Anastomosis site leakage & $31(72.1 \%)$ \\
\hline Cut surface leakage & $2(4.7 \%)$ \\
\hline Hepatocyte dysfunction & $3(6.9 \%)$ \\
\hline \multicolumn{2}{|l|}{ Intervention } \\
\hline No intervention & $9(20.9 \%)$ \\
\hline ERCP & $15(34.8 \%)$ \\
\hline PTBD & $6(13.9 \%)$ \\
\hline PCD & $13(30.2 \%)$ \\
\hline In-hospital diagnosis of biliary leakage & $22(51.2 \%)$ \\
\hline Readmission and diagnosis & $21(48.8 \%)$ \\
\hline
\end{tabular}

*Every recipient underwent abdominal $\mathrm{CT}$ at postoperative 7 days

HBV LC, hepatitis-B related liver cirrhosis; HCV LC, hepatitis-C related liver cirrhosis; ERCP, endoscopic retrograde cholangiopancreatography; PTBD, percutaneous transhepatic biliary drainage 
low-up computed tomography (CT) or when the drainage color was thick green. Moreover, in case of persistent hyperbilirubinemia because of graft dysfunction due to chronic rejection or recurrent cholangitis tests, MR cholangiography with Gd-EOB-DTPA was performed to evaluate the liver function. Herein, we have classified the purpose and results of MR Cholangiography with Gd-EOB-DTPA and described its functionality in each case.

\section{MR cholangiography with Gd-EOB-DTPA with 40-minute delay protocol}

MR examinations were performed on a $1.5-\mathrm{T}$ whole-body MR scanner (SignaHDx; GE Healthcare, Milwaukee, WI) with an eight-channel torso phased-array coil. All images were obtained either in the axial or coronal plane. GdEOB-DTPA (Primovist ${ }^{\circledR}$, Bayer-SheringPharma, Berlin, Germany) was administered at a dose of $0.025 \mathrm{mmol} / \mathrm{kg}$ $(0.1 \mathrm{ml} / \mathrm{kg}$ body weight $)$ at a rate of $1.5 \mathrm{ml} / \mathrm{s}$ followed by a 30-ml saline flush. Using an MR fluoroscopic technique, hepatic arterial phase images were obtained $9 \mathrm{sec}$ after the arrival of contrast medium at the descending thoracic aorta, after which two additional sequential axial image sets were obtained at 10 -second intervals during the first minute after the hepatic arterial phase, thereby obtaining both portal phase and hepatic venous phase images. Thereafter, late dynamic phase and hepatobiliary phase images were then obtained at 3,10, 20, and 40 minutes, respectively, following the contrast injection.

\section{RESULTS}

We have summarized the demographic data of the recipients who underwent the T1-Weighted MR Cholangiography with Gd-EOB-DTPA in Table 1. The average age of the recipients was $51 \pm 12.9$ years and $34(79.1 \%)$ were men and 9 (20.9\%) were women. In 19 (44.2\%) cases Hepatitis B related cirrhosis was the most common indication for LT, alcoholic liver cirrhosis was 14 cases (35.6\%), and re-transplantation due to chronic rejection was 2 cases $(4.7 \%)$. Forty-one patients $(95.3 \%)$ underwent LDLT with the right liver, 1 case $(2.3 \%)$ of LDLT using the left liver, and 1 case $(2.3 \%)$ of the deceased donor liver transplantation using the whole liver.

From the radiologic examinations performed in patients suspected of bile duct complication, 95\% (38/40) of the patients had bile leakage and stricture. Cut surface leakage was diagnosed in two cases, and the other cases were diagnosed with biliary leakage from the anastomosis site. Most patients (29/38, 76.3\%) with bile duct complications underwent percutaneous drainage and ERCP.

It was used for checking the bile secretion function of the hepatocyte. There was no contrast-enhanced bile duct image in 2 cases with severe rejection, which may have been related to hepatocyte secretary dysfunction.

\section{Anastomosis leakage evaluation}

Forty patients had undergone MR Cholangiography with Gd-EOB-DTPA due to suspected biliary complications. Biloma was observed during routine follow-up CT in 10 cases (23.3\%), and 11 cases $(25.6 \%)$ in whom the drainage color had changed to greenish and 14 cases $(32.5 \%)$ of biloma confirmed by $\mathrm{CT}$ at the emergency department due to abdominal pain. Anastomosis leakage was confirmed in 33 cases of the 37 suspected patients with leakage. Fifteen (34.8\%) patients underwent endoscopic retrograde biliary drainage (ERBD) through ERCP, and six (13.9\%) patients experienced ERCP failure and subsequently underwent percutaneous transhepatic biliary drainage (PTBD) (Fig. 1). Thirteen patients (30.2\%) with large-sized biloma on CT taken with symptoms were visualized on MRI Cholangiography with Gd-EOB-DTPA after reducing the biloma cavity after percutaneous catheter drainage (PCD) insertion (Fig. 2). In the 20 min delay image, anastomosis can be well evaluated, and in the $40 \mathrm{~min}$ delay image, the contrast agent can confirmed the site of leakage.

Among 38 patients with confirmed leakage or stricture, 37 were found to have leakage or stricture on MRI and confirmed positive results. The sensitivity for bile-duct complication detection was $97.3 \%$ (37/38). The number of patients without biliary complication was two and no biliary complication was identified from GD-EOG-MRI in all of these patients. The specificity of the test was $100 \%$ $(2 / 2)$ (Table 2). The connection between biloma and CBD was confirmed in the PCD tubogram in one patient among three who showed negative findings on MRI.

Differential diagnosis of cut surface leakage

Among the patients suspected with biliary complication, two were diagnosed with the leakage of the cut surface, not of the anastomosis site. The PCD was placed into the biloma cavity in the two cases and the amount of drainage was decreased. The two patients were discharged 

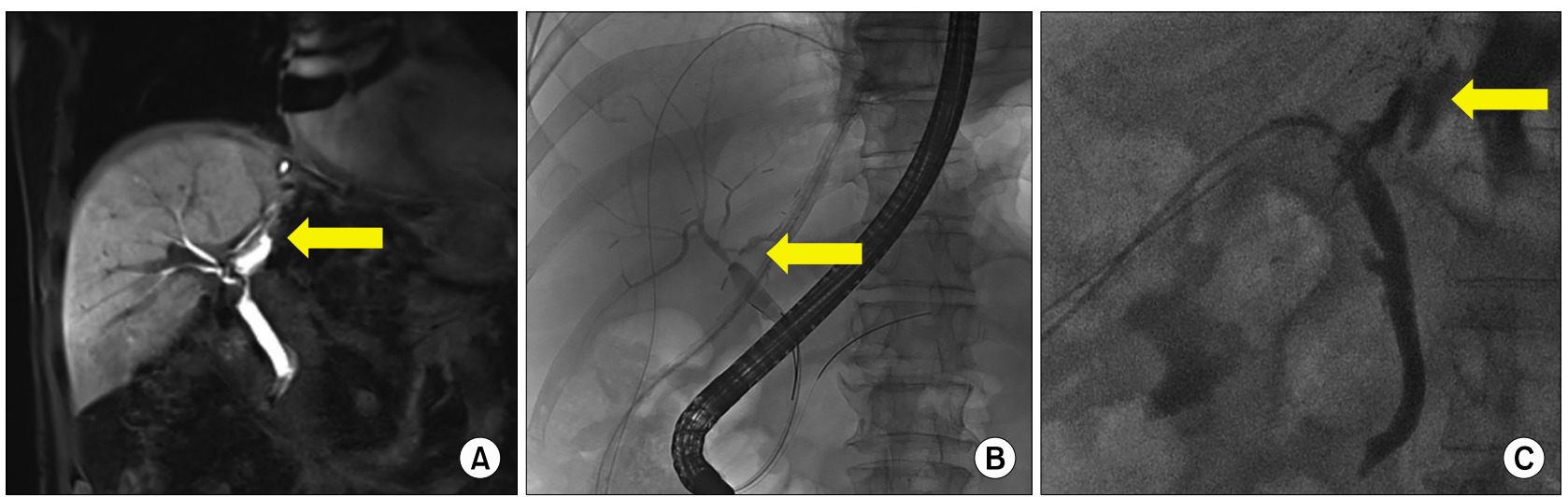

Fig. 1. (A) Gd-EOB DTPA 40 minute delay image in the case of biliary anastomosis leakage. (B) ERCP findings in the case of biliary anastomosis leakage. (C) PTBD findings in the case of biliary anastomosis leakage.
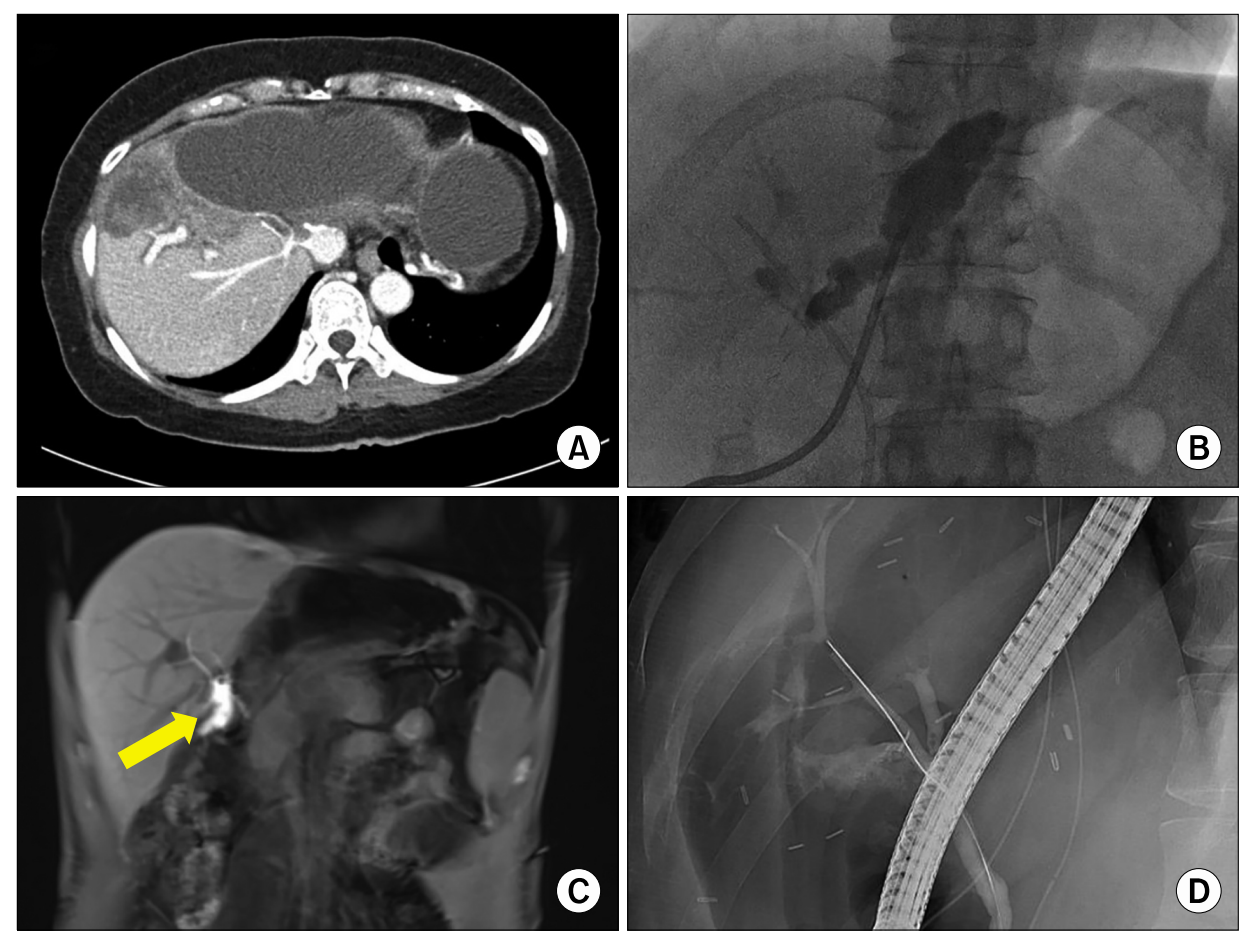

Fig. 2. (A) Incidentally found biloma in the postoperative 7 days CT after LT. (B) CT finding after percutaneous drainage $(\mathrm{PCD})$ in the biloma. (C) Gd-EOB DTPA MRI findings of leakage site after PCD drainage. (D) Interventional fluoroscopy for ERCP/PTBD.

after conservative management. The case was a 62-year-old man who had a Jackson-Pratt drain color change after LDLT. A biloma was identified around the liver cut surface on the postoperative 7 days abdominal CT scan (Fig. 3). The PCD was placed into the biloma cavity thereafter MR Cholangiography with Gd-EOB-DTPA with a $40 \mathrm{~min}$ delay image was performed for the differential diagnosis of biliary complication. From the MR Cholangiography, no direct connection from the anastomosis to biloma was noted and the distance between biloma and the anastomosis site was a little more. This case was diagnosed as a cut surface leakage, percutaneous drainage without ERCP or PTBD was performed. The patient's condition improved after drainage.

Evaluation of liver dysfunction

MR Cholangiography with Gd-EOB-DTPA was performed to evaluate the liver excretion in 3 cases with recurrent cholangitis due to bile duct complication or suspected liver dysfunction due to chronic rejection. Three cases had liver parenchymal damage due to chronic rejection and recurrent cholangitis, and one of the patient was admitted for acute onset hyperbilirubinemia on the liver function test in the outpatient clinic. Abdominal CT was performed to evaluate the structural defect of the bile 
Table 2. Sensitivity and specificity of the Gd-EOB DTPA MRI 40 minute delay protocol for diagnosis of biliary complication after LT

\begin{tabular}{lcc}
\hline & Biliary complication & No biliary complication \\
\hline Gd-EOB-MRI with a 40 min delay Positive & 37 & 0 \\
Gd-EOB-MRI with a 40 min delay Negative & 1 & 2 \\
Sensitivity/specificity & Sensitivity $97.3 \%(37 / 38)$ & Specificity $100 \%(2 / 2)$ \\
\hline
\end{tabular}

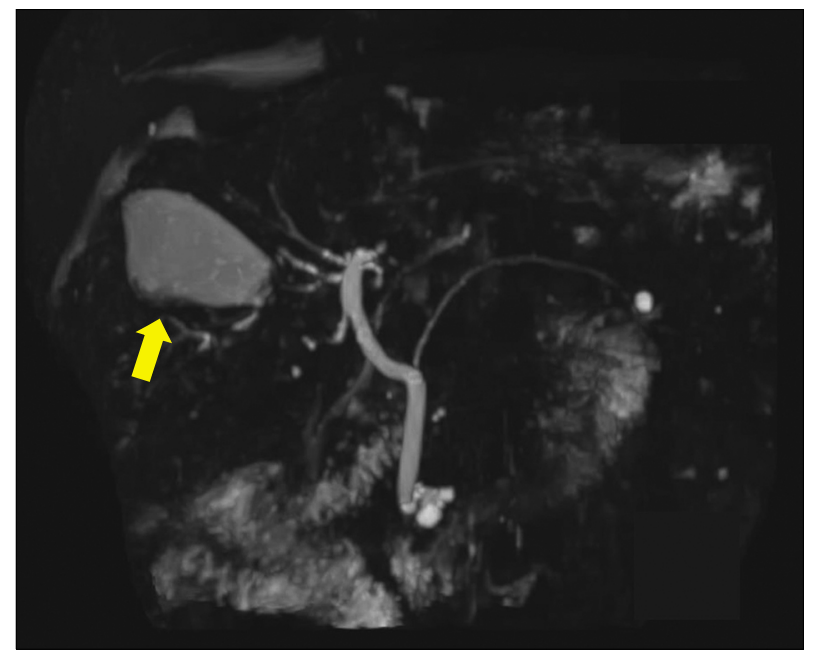

Fig. 3. Gd-EOB DTPA MRI findings of the case with cut surface leakage.

duct, but the bile duct was not dilated. The result of the liver biopsy performed for the differential diagnosis of hyperbilirubinemia revealed no acute cellular rejection but confirmed the chronic rejection. MR Cholangiography with Gd-EOB-DTPA was performed to confirm hyperbilirubinemia caused by dysfunction of bile secretion due to chronic change. In a normal case, bile duct is usually observed in the $20 \mathrm{~min}$ delay image. However, the bile duct was not observed from the MR Cholangiography with Gd-EOB-DTPA even after a 40 min delay (Fig. 4). It was related to the dysfunction of the secretion and bile production. The bile transporter could be damaged due to chronic liver damage caused by rejection and recurrent cholangitis.

\section{DISCUSSION}

Gd-EOB-DTPA-enhanced MRI is an alternative technique to evaluate the biliary tree according to its biliary excretion. $^{10,12,15}$ It has been utilized for the preoperative workup of potential living liver donors in the field of LT. Except in some cases with organic anion transporting pol-

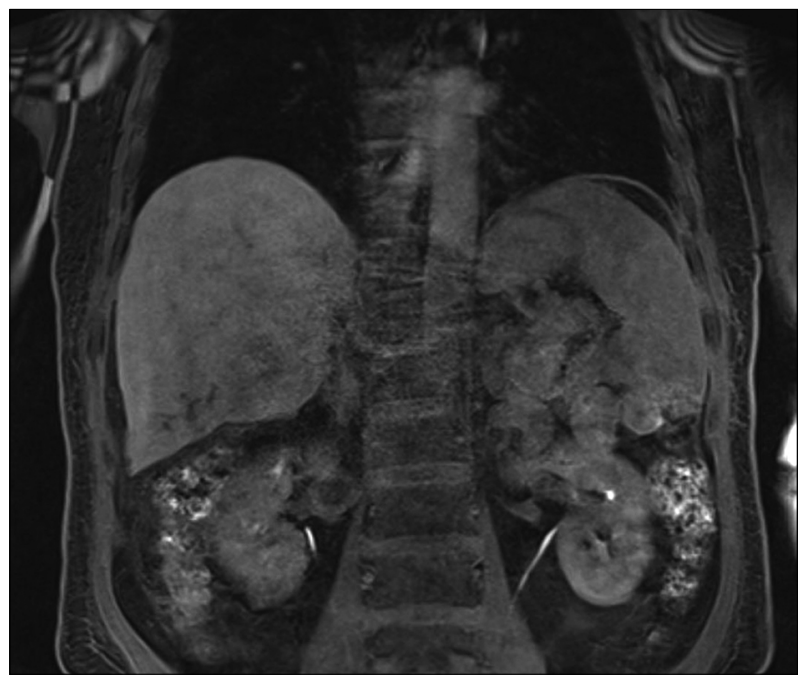

Fig. 4. GD-EOB DTPA MRI findings of the case with chronic rejection. No bile secretion was identified in the biliary tract.

ypeptide expression disorder in the donor, it demonstrated good or excellent agreement for visualizing the biliary tree even in a case with a complex anatomical variation.

Meanwhile, several attempts to noninvasively confirm biliary leakages, such as MRCP or hepatobiliary scan (DISIDA scan), have continued. Previous reports have revealed the usefulness of scans in the identification of leakage in hepatobiliary surgery, as well as research on images obtained using MRCP.

However, the two imaging tools were indeed difficult to accurately read because the image blurring was quite severe. DISIDA scans are functional studies of the gallbladder. Technetium-labeled analogs of iminodiacetic acid (IDA) or diisopropyl IDA-DISIDA are administered intravenously and are secreted by the hepatocytes into the bile, enabling the visualization of the liver and biliary tree. ${ }^{9,16}$ Although bile secretion can be confirmed, it is difficult to observe the surrounding structures accurately, and efforts were made to overcome this problem through fusion images with SPECT-CT. ${ }^{17}$ However, since it fuses two different image tools, it is inferior to MR cholangiography 
with GD-EOB-DTPB aspect of the accurate localization.

Magnetic resonance cholangiopancreatography (MRCP) is not a functional imaging modality, it is an imaging method to confirm the structure, and the structure also has blurring compared to $\mathrm{CT}$, and therefore it is not a favorable tool to observe the functional images such as leakage. MR cholangiography with Gd-EOB-DTPA can be considered to have the advantages of both the methods. By using a contrast agent, it is possible to obtain a slightly clearer, sequential, and functional image, which has the greater advantage of enabling the simultaneous evaluation of the structure and function with one tool. Moreover, as the leakage point is the cut surface rather than the anastomosis, differentiation is easy as shown in Fig. 2, reducing unnecessary procedures.

If the bile duct was not observed in the Gd-EOB DTPA MRI 40-minute delay image, the main cause of the hyperbilirubinemia was liver dysfunction. When liver dysfunction is identified, it helps to avoid insignificant bile duct intervention and reconfirm long-term strategies. ERCP or PTBD procedures can cause problems such as bleeding and pancreatitis. In the immediate postoperative period after LT, coagulopathy sometimes occurs and can cause major complications. Additionally, in the case of cut surface leakage, diagnosis with ERCP alone is difficult, and leakage sites may not be identified without accurate cannulation technique; therefore, it will be helpful for the interventionist to proceed with an intervention if the leakage point can be predicted and guided using the MRI.

The limitation of this study is that most of the patients diagnosed with complications finally underwent biliary intervention. Therefore, one may consider if the procedure is justified. Additionally, because the cost of this test does not allow its frequently use, there can be a debate about the procedure when the anastomosis leakage is obvious.

However, if unnecessary ERCP or biopsy procedures can be reduced through the confirmation of cut surface leakage or liver dysfunction, there might be an advantage in considering the cost and risk aspects. Depending on the insurance coverage in each country and whether or not the inspection is set, application at all transplant centers may be difficult. However, we consider its relevance as sufficient in that the most accurate and clean images can be obtained as a tool for diagnosing noninvasive bile duct problems after the previously reported hepatobiliary surgery.
In conclusion, MR cholangiography with GD-EOB-DTPB 40-min delay image is a useful, safe, and non-invasive diagnostic tool for the evaluation of bile duct complication and liver dysfunction after LT. It can not only show a clear reconstruction image but also its functional status. Although it has a cost and insurance coverage problem, we consider it as favorable in terms of reducing unnecessary repetitive invasive procedures through accurate diagnosis without any complication related examination.

\section{CONFLICT OF INTEREST}

The authors have no conflicts of interest to declare.

\section{ORCID}

Chang Jin Lim: https://orcid.org/0000-0002-4263-7910 Kwangpyo Hong: https://orcid.org/0000-0002-3220-8506 Jeong-Moo Lee: https://orcid.org/0000-0001-7806-8759

Eui Soo Han: https://orcid.org/0000-0001-9582-1255

Suk Kyun Hong: https://orcid.org/0000-0002-0020-6215 YoungRok Choi: https://orcid.org/0000-0003-2408-7086 Nam-Joon Yi: https://orcid.org/0000-0002-5467-425X Kwang-Woong Lee: https://orcid.org/0000-0001-6412-1926 Kyung-Suk Suh: https://orcid.org/0000-0002-9535-7349

\section{AUTHOR CONTRIBUTIONS}

Conception and design: JML. Acquisition, analysis, or interpretation of data: All authors. Drafting of the manuscript: CJL, KH. Critical revision of the manuscript for important intellectual content: JML, SKH, YC, NJY, KWL, KSS. Statistical analysis: CJL, JML, KH, ESH. Administrative, technical, or material support: NJY, KWL. Supervision: JML, NJY, KWL, KSS.

\section{REFERENCES}

1. Woo HY, Lee IS, Chang JH, Youn SB, Bae SH, Choi JY, et al. Outcome of donor biliary complications following living donor liver transplantation. Korean J Intern Med 2018;33:705-715.

2. Nakamura T, Iida T, Ushigome H, Osaka M, Masuda K, Matsuyama $\mathrm{T}$, et al. Risk factors and management for biliary complications following adult living-donor liver transplantation. Ann Transplant 2017;22:671-676.

3. Chang JH, Lee I, Choi MG, Han SW. Current diagnosis and treatment of benign biliary strictures after living donor liver transplantation. World J Gastroenterol 2016;22:1593-1606. 
4. Axelrod DA, Lentine KL, Xiao H, Dzebisashvilli N, Schnitzler M, Tuttle-Newhall JE, et al. National assessment of early biliary complications following liver transplantation: incidence and outcomes. Liver Transpl 2014;20:446-456.

5. Hwang S, Lee SG, Sung KB, Park KM, Kim KH, Ahn CS, et al. Long-term incidence, risk factors, and management of biliary complications after adult living donor liver transplantation. Liver Transpl 2006;12:831-838.

6. Kim JM, Cho W, Kwon CHD, Joh JW, Park JB, Ko JS, et al. Bile duct reconstruction by a young surgeon in living donor liver transplantation using right liver graft. Medicine (Baltimore) 2014;93:e84.

7. Suh KS, Suh SW, Lee JM, Choi Y, Yi NJ, Lee KW. Recent advancements in and views on the donor operation in living donor liver transplantation: a single-center study of 886 patients over 13 years. Liver Transpl 2015;21:329-338.

8. Eckenschwiller M, Ackermann H, Bechstein WO, Grünwald F. Accuracy of hepatobiliary scintigraphy after liver transplantation and liver resection. Int J Mol Imaging 2016;2016:7857849.

9. de Graaf W, Bennink RJ, Veteläinen R, van Gulik TM. Nuclear imaging techniques for the assessment of hepatic function in liver surgery and transplantation. J Nucl Med 2010;51:742-752.

10. Cai L, Yeh BM, Westphalen AC, Roberts J, Wang ZJ. 3D T2-weighted and Gd-EOB-DTPA-enhanced 3D T1-weighted MR cholangiography for evaluation of biliary anatomy in living liver donors. Abdom Radiol (NY) 2017;42:842-850.

11. Boraschi P, Donati F, Gigoni R, Filipponi F. Biliary complica- tions following orthotopic liver transplantation: may contrast-enhanced MR Cholangiography provide additional information? Eur J Radiol Open 2016;3:108-116.

12. Xu YB, Bai YL, Min ZG, Qin SY. Magnetic resonance cholangiography in assessing biliary anatomy in living donors: a meta-analysis. World J Gastroenterol 2013;19:8427-8434.

13. Boraschi P, Donati F, Gigoni R, Volpi A, Salemi S, Filipponi $\mathrm{F}$, et al. MR cholangiography in orthotopic liver transplantation: sensitivity and specificity in detecting biliary complications. Clin Transplant 2010;24:E82-E87.

14. Boraschi P, Braccini G, Gigoni R, Sartoni G, Neri E, Filipponi F, et al. Detection of biliary complications after orthotopic liver transplantation with MR cholangiography. Magn Reson Imaging 2001;19:1097-1105.

15. Kul M, Erden A, Düşünceli Atman E. Diagnostic value of GdEOB-DTPA-enhanced MR cholangiography in non-invasive detection of postoperative bile leakage. Br J Radiol 2017;90: 20160847.

16. Kim YJ, Lee KT, Jo YC, Lee KH, Lee JK, Joh JW, et al. Hepatobiliary scintigraphy for detecting biliary strictures after living donor liver transplantation. World J Gastroenterol 2011;17: 2626-2631.

17. Arun S, Santhosh S, Sood A, Bhattacharya A, Mittal BR. Added value of SPECT/CT over planar Tc-99m mebrofenin hepatobiliary scintigraphy in the evaluation of bile leaks. Nucl Med Commun 2013;34:459-466. 